\title{
White Dwarf Pulsars as Possible Gamma-Ray Sources
}

Q. Kaplan ${ }^{1}$, P.J. Meintjes ${ }^{1 *}$, K.K. Singh ${ }^{1,2}$, H.J. van Heerden ${ }^{1}$, F.A. Ramamonjisoa ${ }^{1}$

1.) Department of Physics, University of the Free State, PO Box 339, Bloemfontein, 9300

2.) Astrophysical Sciences Division, Bhaba Atomic Research Centre, Mumbai, India, 400085

E-mail: MeintjPJ@ufs.ac.za

The newly discovered white dwarf binary system AR Scorpii (AR Sco) with its fascinating nonthermal dominated multi-frequency emission has sparked renewed interest in potential high energy gamma-ray emission from white dwarf pulsars. Recent studies revealed that the optical and X-ray components of the emission in AR Sco can be powered entirely by the spin-down luminosity of the white dwarf in the absence of any mass accretion in this system. The Spectral Energy Distribution (SED) below and above optical shows a clear indication of a non-thermal synchrotron component, with pulsed emission in optical and X-ray bands at the white dwarf spin period as well as a beat with the binary period. From an energy perspective the highly magnetic rotating white dwarf can accelerate particles to $\mathrm{TeV}$ energies. In this study, a search for high energy gamma-ray emission was conducted between $100 \mathrm{MeV}-500 \mathrm{GeV}$ by analysing the newly available Fermi-LAT Pass 8 data with the new Fermi 1.0.1 Science Tools. From the selected Region of Interest (ROI) centred on AR Sco's position we calculated a significance of $\sqrt{T S} \leq 4.89 \sigma$ for the integrated gamma-ray activity between $100 \mathrm{MeV}-500 \mathrm{GeV}$ at a photon flux level of $1.076 \pm 0.318 \times 10^{-8}$ photons $\mathrm{cm}^{-2} \mathrm{~s}^{-1}$. However, the close-by unidentified source $\left(\sim 0.576^{\circ}\right) 4 F G L J 1623.7-2315$ may also contribute to this emission. AR Sco's location inside the Rho Ophiuchi (Rho Oph) molecular cloud complex combined with the poor spatial resolution of Fermi-LAT complicates any unambiguous identification of the source of low-level gamma-ray activity at the location that coincides with the position of AR Sco.

High Energy Astrophysics in Southern Africa - HEASA2018

1-3 August, 2018

Parys, Free State, South Africa

${ }^{*}$ Speaker. 


\section{Introduction}

The recent discovery ([1]) of the peculiar close binary system AR Scorpii (AR Sco) which displays non-thermal dominated multi-frequency emission (see Fig. 1) from radio to X-rays sparked enormous interest in the astronomy community. The notion that nearly the entire Spectral Energy Distribution (SED) may be dominated by non-thermal emission is of huge theoretical interest (e.g. $[2,3])$. A favorite approach was to model the emission as being the result of the interaction between a highly magnetized white dwarf pulsar and an M-type secondary dwarf star. It also sparked renewed interest in white dwarf pulsars being potential high energy gamma-ray emitters (e.g. [4, 5]). This system consists of a $0.8-1.29 M_{\odot}$ highly magnetic white dwarf with a surface magnetic field that can be as high as $500 \mathrm{MG}$ (e.g. [4, 5]) orbiting an M5 dwarf star with mass ranging between $0.28-0.45 M_{\odot}$ with an orbital period of $P_{\text {orb }}=3.56$ hours. Assuming the secondary star fills its Roche lobe, the distance to the system is estimated to be approximately $d \sim 116 \mathrm{pc}$ [1].

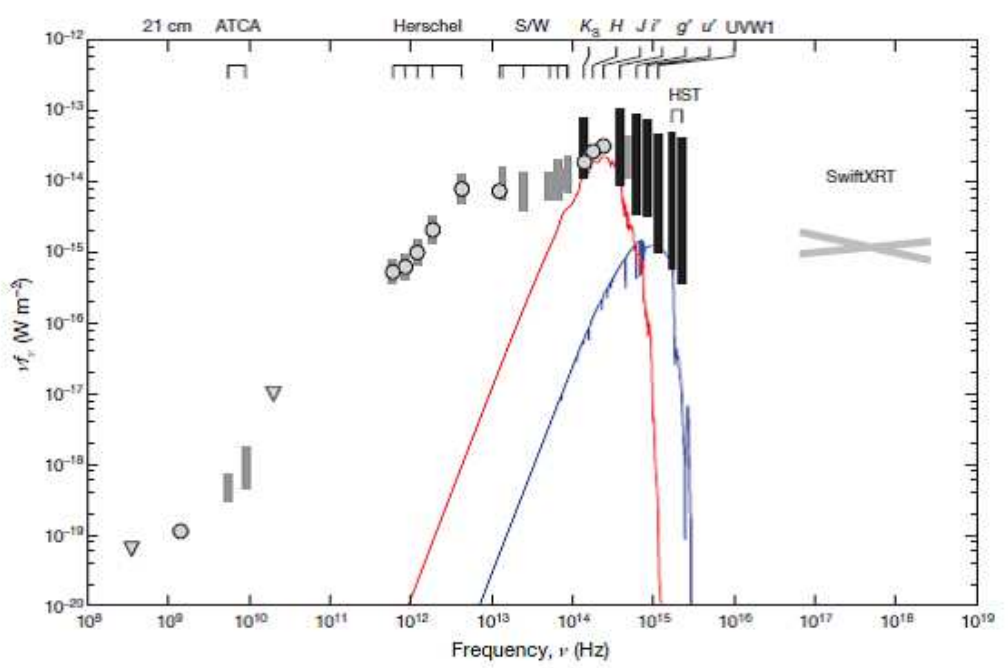

Figure 1: The multi-frequency Spectral Energy Distribution (SED) of AR Scorpii from radio to X-ray frequencies. The red and blue curves represent model atmospheres of the red M5 secondary dwarf $\operatorname{star}(\mathrm{T}=$ $3100 \mathrm{~K})$ and white dwarf $(\mathrm{T}=9750 \mathrm{~K})$ respectively. Adopted from [1].

In the optical band (see [1]) the system reveals pulsations at $117 \mathrm{~s}$, which is the spin period of the white dwarf, superimposed on the orbital period as well as strong pulsations at $118.2 \mathrm{~s}$, which is a beat period between the spin period and the binary orbital period (see Fig. 2). Both the spin and beat periods are clearly visible on a power spectrum (see Fig. 3), especially at the first harmonic where a clear differentiation can be distinguished between the spin and beat periods [1].

High-speed all-Stokes optical polarimetry utilizing the South African Astronomical Observatory (SAAO) HIPPO Polarimeter [6] on the $1.9 \mathrm{~m}$ telescope reveals strong a linear polarization at levels up to $40 \%$ with significantly lower levels of circular polarization $(<10 \%)[4,5]$. The nature of the linear polarization profile, especially the 360 degrees swing in the position angle is consistent with synchrotron emission produced in the magnetic field of a rotating magnetic dipole, strengthening the notion that the white dwarf in AR Sco is a white dwarf pulsar. 


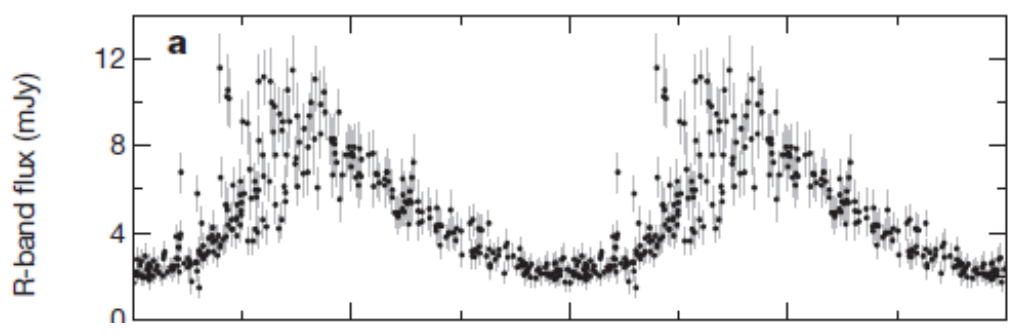

Figure 2: The orbital modulation from AR Scorpii in R-band, showing ellipsoidal variations that may be due to a Roche lobe filling (or nearly lobe filling) secondary star. Superimposed on this orbital light curve, optical pulsations can be seen at the spin period (117 s) and beat period (118.2 s) with the binary system. Adopted from [1].

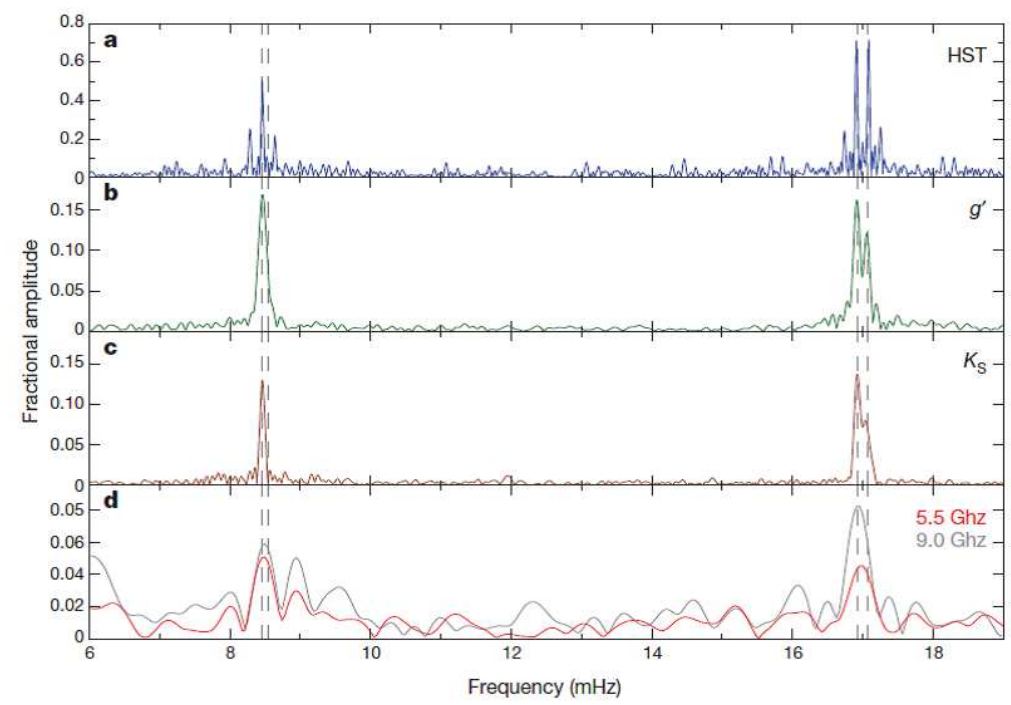

Figure 3: Periodograms from radio to optical (HST), all revealing the strong double pulse beat period between the spin and orbital periods of the system. The beat pulse and the spin frequency are most prominent at the first harmonic, close to $17 \mathrm{mHz}$. The peak on the left represents the beat while the peak on the right represents the spin frequency. Adopted from [1].

Initial pulse timing studies after its discovery, over a baseline of 7 years, suggest that the white dwarf is slowing down, resulting in a frequency derivative of $-\dot{v}_{\mathrm{s}, \mathrm{wd}}=2.86 \times 10^{-17} \mathrm{~Hz} \mathrm{~s}^{-1}$, which for a $\sim 0.8 M_{\odot}$ white $\operatorname{dwarf}\left(R_{\mathrm{wd}} \approx 0.01 R_{\odot}\right)$, infer a spin-down luminosity of approximately $\left.L_{\dot{v}_{\text {s,wd }}} \approx 1.5 \times 10^{33} \mathrm{erg} \mathrm{s}^{-1}[1]\right)$. The result of a more recent study of the spin-evolution of the white dwarf [7] is inconsistent with any reported spin-down, while another study [8] shows an evidence of a spin-down rate through a beat frequency derivative $-\dot{v}_{\mathrm{s}, \mathrm{b}}=5.14 \times 10^{-17} \mathrm{~Hz} \mathrm{~s}^{-1}$ with resultant spin-down power of $L_{\dot{\mathrm{v}}_{\mathrm{s} \text { wd }}} \approx 3 \times 10^{33} \mathrm{erg} \mathrm{s}^{-1}$ which is approximately twice the value reported earlier [1].

Recent studies of the pulsed nature of the emission in X-rays using XMM-Newton data [10] reveal pulsed emission consistent with the white dwarf spin period with a pulsed fraction of $\sim$ 
$14 \%$. These authors suggest that the X-ray emission is produced mainly by a thermal plasma on the secondary star heated by magnetic pumping from the white dwarf field that sweeps across it during every rotation cycle. The X-ray luminosity of $L_{x}=4 \times 10^{30} \mathrm{erg} \mathrm{s}^{-1}$ is then produced mainly by the thermalized electrons on the face of the secondary star, with the non-thermal component, peaking in IR/Optical, produced by relativistic electrons accelerated in the magnetosphere of the white dwarf $[9,10]$. Further, they propose that the emission is consistent with a power-law electron spectrum with index ranging between $\mathrm{p}=2.5-3$.

For AR Sco the ratio of the X-ray luminosity to spin-down power, i.e. $\alpha=L_{x} / L_{\dot{v}_{\mathrm{s}, \mathrm{wd}}} \sim 10^{-3}$, is similar to another cataclysmic variable AE Aquarii and place both AR Sco and AE Aquarii in the same class as spin-powered pulsars [11] (see Fig. 4). The high ratio of spin-down power to thermal X-ray emission provides a reservoir for particle acceleration that makes AR Sco a possible candidate for high energy gamma-ray emission like most other spin-powered pulsars [11].

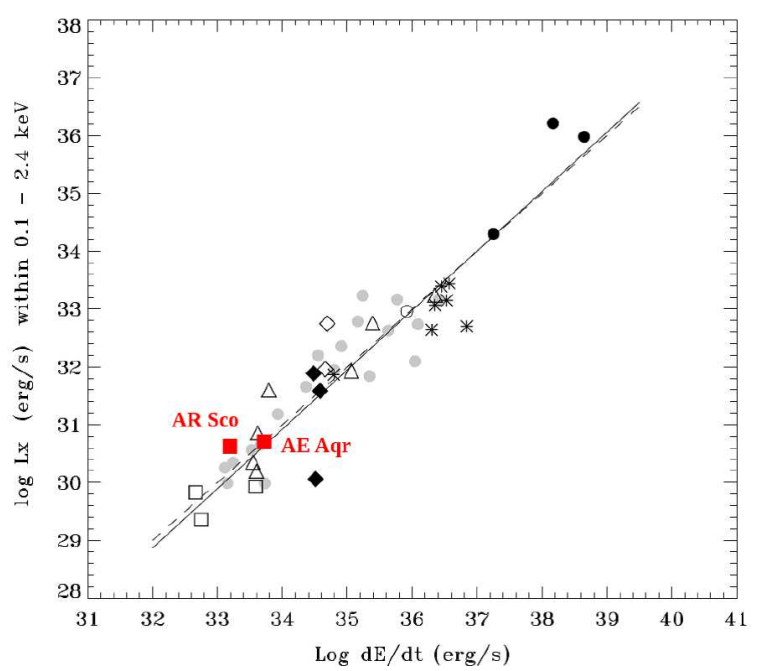

Figure 4: The X-ray luminosity versus spin-down power of spin-powered neutron stars, compared to both the white dwarfs in AR Sco and AE Aquarii. Adapted from [11].

\section{The search for gamma-ray emission using 10 years of Fermi-LAT data}

A Fermi-LAT dataset from the past decade (4 August 2008 - 18 March 2019) was extracted from the Fermi Science Support Center (FSSC) in the energy range of $100 \mathrm{MeV}$ to $500 \mathrm{GeV}$. By using the Pass 8 data analysis pipeline, which provides a better determination of the diffuse galactic gamma-ray emission and a significant improvement in terms of energy resolution from previous Fermi-LAT pipelines, it was possible to do a standard Binned Likelihood Analysis on the Fermi-LAT dataset centred on the position of AR Sco. The analysis was performed using the recently released Fermi Tools software (Fermi 1.0.1), where a number of energy ranges were experimented with to fit and produce the likelihood statistics for AR Sco. The event files, i.e. the photon and spacecraft files that were extracted from the FSSC site, were chosen to have a Region of Interest (ROI) of $10^{\circ}$ centred on AR Sco's position (RA: $16^{h} 21^{m} 47.28^{s}$, Dec: $-22^{\circ} 53^{\prime} 10.39^{\prime \prime}$, J2000) in order to extract the high energy photons for analysis. During reduction, only SOURCE 
class events (evclass $=128$ ) were considered, along with the relational filter (DATA_QUAL $>0$ and LAT_CONFIG $==1$ ) to select good time intervals for the period 4 August 2008 - 18 March 2019.
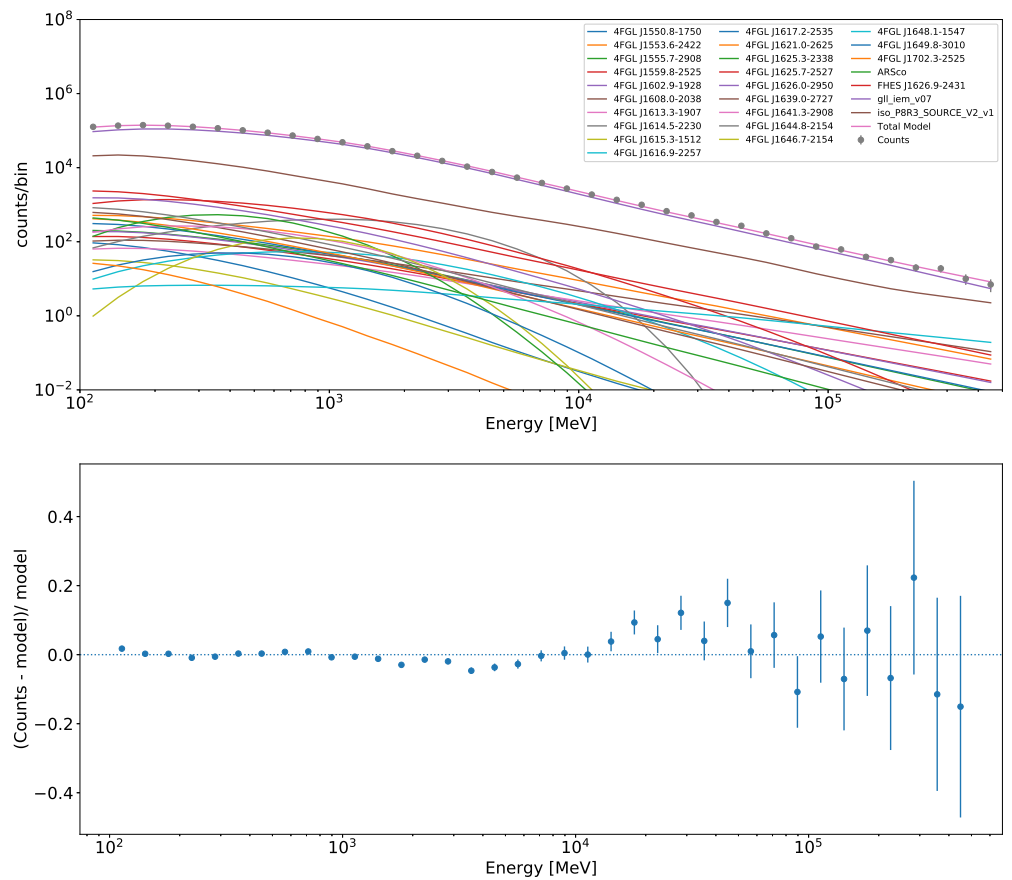

Figure 5: Counts model (top) and residual fit (bottom) from the binned likelihood analysis for AR Sco using power law spectral shape in the energy range $100 \mathrm{MeV}$ to $500 \mathrm{GeV}$.

All the point sources in the fourth Fermi-LAT catalogue (4FGL) located within the ROI were used to generate the background model including the latest isotropic background and galactic diffuse emission. The spectral forms of all the point sources included in the background model file are the same as reported in the 4FGL catalogue. As AR Sco is not listed in the 4FGL catalogue, we have followed the same methodology to perform the binned likelihood analysis as ([12]) which reported the detection of a gamma-ray flare from the high redshift blazar DA 193. The target source AR Sco was added in the model file with a power law spectral shape. The associated parameters of spectral models of all the sources within the ROI were chosen to be free to vary while performing the binned likelihood fitting. The prefactor and photon index of AR Sco were also allowed to vary during the fitting. The significance of the $\gamma$-ray signal was determined from the maximum likelihood test statistic (TS) which follows a $\chi^{2}$ distribution with two degrees of freedom, resulting in an upper limit significance $(\sigma)$ for a large number of data points which can be approximated by $\sqrt{T S}([13,14])$. All the sources, except AR Sco, with a TS value of less than 25 were removed 
from the model file and the likelihood fitting was re-performed using these sources. The fitting was repeated to optimize the background model file with sources having a TS value more than or equal to 25 . The results of the final binned likelihood fitting for modelling the counts and corresponding residual fit in the energy range $100 \mathrm{MeV}$ to $500 \mathrm{GeV}$ are shown in Fig. 5, indicating convergence up to an energy value of $10 \mathrm{GeV}$. The values of the model parameters derived from the fitting for AR Sco in different energy bands are summarized in Table 1. The model parameters reported in Table 1 indicate that the $\gamma$-ray emission from the position of AR Sco in the energy range $100 \mathrm{MeV}$ $500 \mathrm{GeV}$ is mainly dominated by the photons with energy in lower energy band $100 \mathrm{MeV}-10 \mathrm{GeV}$. Above $10 \mathrm{GeV}$, the $\gamma$-ray emission from AR Sco is below the detection sensitivity of the FermiLAT. The residual map in the absence of a source (see Fig. 6) and TS map (see Fig. 7) seem to suggest an excess of bright pixels at the centre of the ROI. The distribution of TS values as a function of energy (see Fig. 8) also indicates that $\gamma$-ray emission from the direction of AR Sco is more significant at lower energies in the range between $100 \mathrm{MeV}$ and $10 \mathrm{GeV}$. However, it is important to note here that an unidentified $\gamma$-ray source namely $4 F G L J 1623.7-2315$ is also present in the background model at an angular separation of $0.576^{\circ}$ from the position of AR Sco (or from the ROI center). Therefore, $\gamma$-ray emission from the position of AR Sco in the energy range $100 \mathrm{MeV}-10$ $\mathrm{GeV}$ detected by the Fermi-LAT can be contaminated by the emission from 4FGLJ1623.7 - 2315 due to its poor angular resolution at low energies. A detailed analysis of the Fermi-LAT data is in progress to properly quantify possible $\gamma$-ray emission from the region containing AR Sco.

Table 1: Summary of model parameters from the power law fit for AR Sco (at the centre of ROI) using Binned Likelihood Analysis for the period 4 August 2008 - 18 March 2019.

\begin{tabular}{lccccc}
\hline Energy Range & Spectral Index & $\mathrm{N}_{\text {pred }}$ & Integral Flux $\left(\mathrm{ph} / \mathrm{cm}^{2} / \mathrm{s}\right)$ & $\mathrm{TS}$ & Significance \\
\hline $100 \mathrm{MeV}-500 \mathrm{GeV}$ & $2.70 \pm 0.82$ & 2041.91 & $(1.076 \pm 0.318) \times 10^{-8}$ & 23.93 & $\leq 4.89 \sigma$ \\
$100 \mathrm{MeV}-10 \mathrm{GeV}$ & $2.81 \pm 0.22$ & 1866.78 & $(0.997 \pm 0.317) \times 10^{-8}$ & 19.35 & $\leq 4.40 \sigma$ \\
$10 \mathrm{GeV}-500 \mathrm{GeV}$ & $6.03 \pm 1.04$ & 0.00 & $(5.681 \pm 1.160) \times 10^{-16}$ & 0 & 0 \\
\hline
\end{tabular}

The results of our present analysis in the energy range between $0.1-500 \mathrm{GeV}$ reveals an excess at the $\sqrt{T S} \leq 4.89 \sigma$ (e.g. [14]) significance level from the position of AR Sco at the centre of the ROI which also contains a nearby unclassified source $4 F G L J 1623.7-2315$. After the likelihood analysis has been performed, SEDs were plotted using the Power Law model over 9 energy bins (see Fig. 9). The SED was obtained using the Fermi build-in function bdlikeSED. The energy flux values can be considered as $2 \sigma$ upper limits. A broadband SED (see Fig. 10) was also created with radio and optical data obtained from ([1]) and X-ray data obtained from ([10]). The flux upper limits from the AR Sco ROI between $100 \mathrm{MeV}$ - $500 \mathrm{GeV}$ coincides with the Fermi-LAT (10 year) [blue curve] threshold but are well below the CTA-South (50 hour) [red curve] sensitivity level in this energy interval.

\section{Conclusions}

A search for possible gamma-ray emission from AR Sco was carried out by analysing the Fermi-LAT Pass 8 dataset using the Fermi Science Tools. The results from a binned-likelihood 


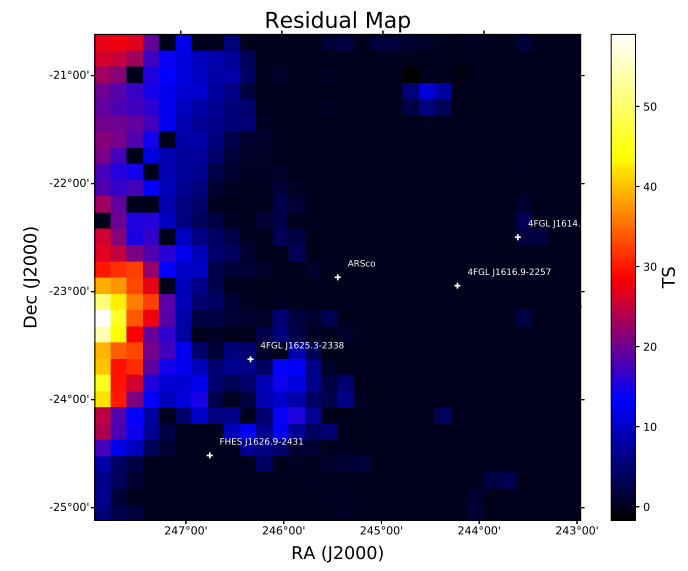

Figure 6: Residual map in the absence of target source at the centre of the ROI.

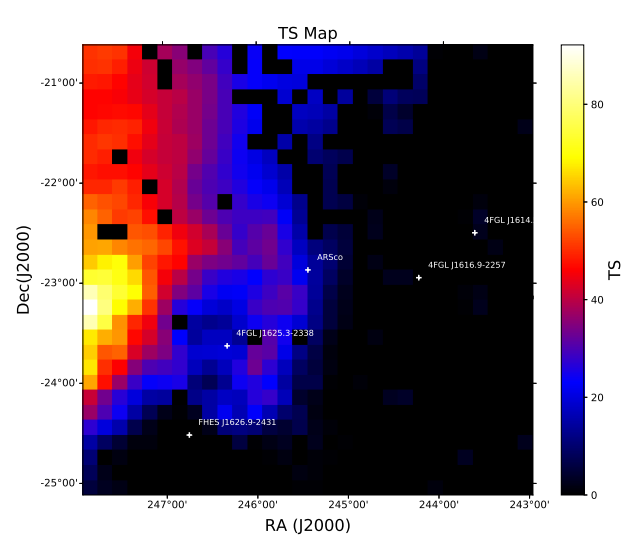

Figure 7: TS map displaying excess of bright pixels at the centre of the ROI.

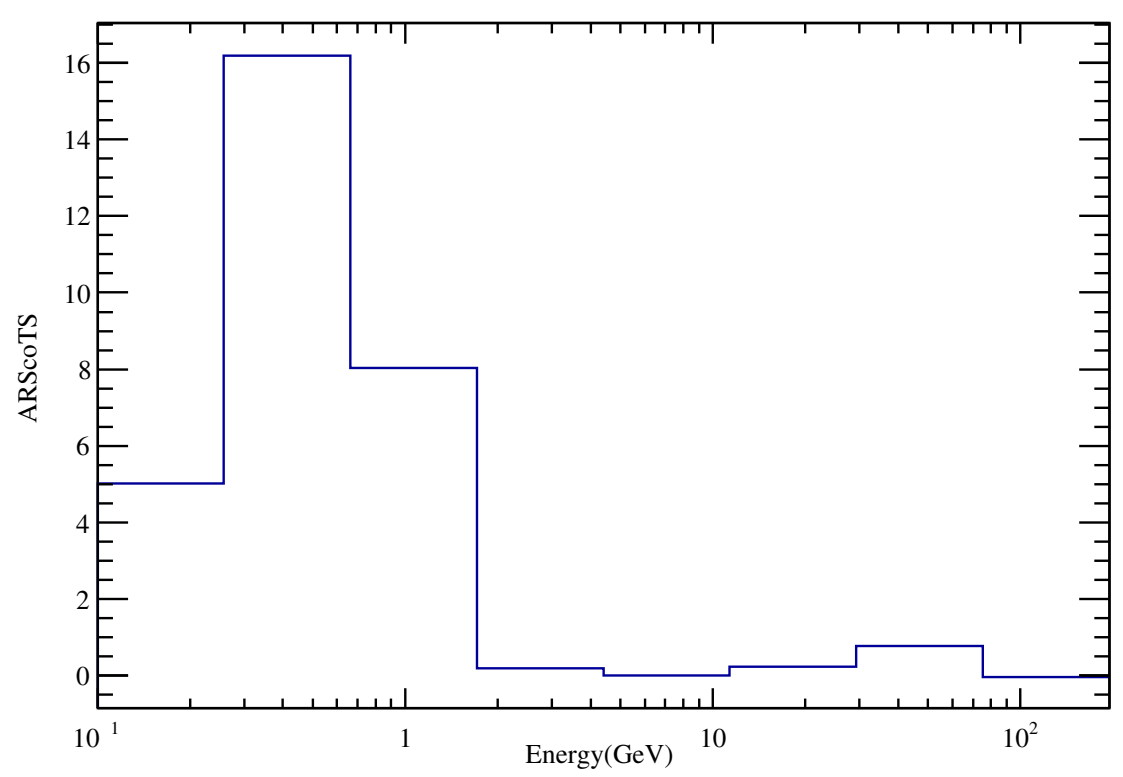

Figure 8: TS values vs energy of $\gamma$-ray photons.

analysis on a ROI with a radius of $10^{\circ}$ centred on the position of AR Sco, optimized with a powerlaw model of index $(\Gamma=-2.7)$, revealed a gamma-ray flux between $100 \mathrm{MeV}-500 \mathrm{GeV}$ at the $\sqrt{T S} \leq 4.89 \sigma$ level. This however may include some emission from an unclassified source $4 F G L J 1623.7-2315$ which contribution to the total emission is still uncertain. The flux upper limits per energy bin correlates with the 10 year Fermi-LAT (blue) threshold but are well below the 50 hour CTA-South (red) sensitivity threshold. The location of AR Sco in the Rho Oph cloud complex combined with the presence of the nearby unclassified 4FGL source makes any unambiguous identification very difficult at this stage. 


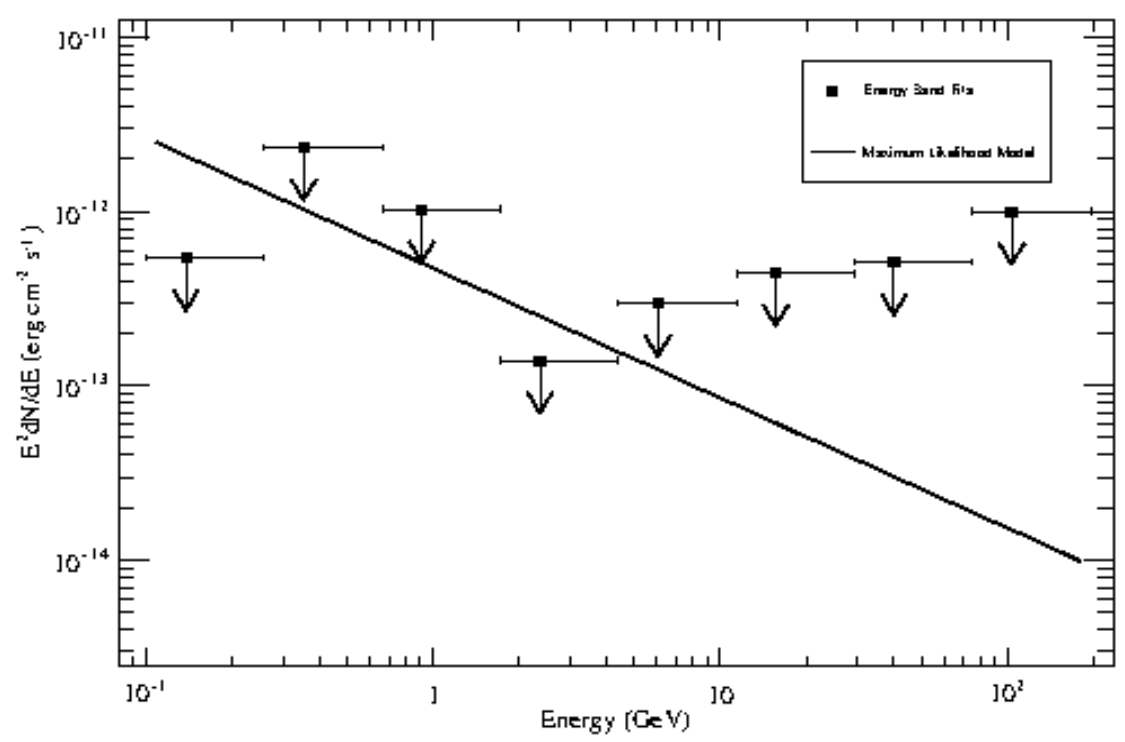

Figure 9: Energy flux SED obtained using bdlikeSED. The flux values are $2 \sigma$ upper limits.

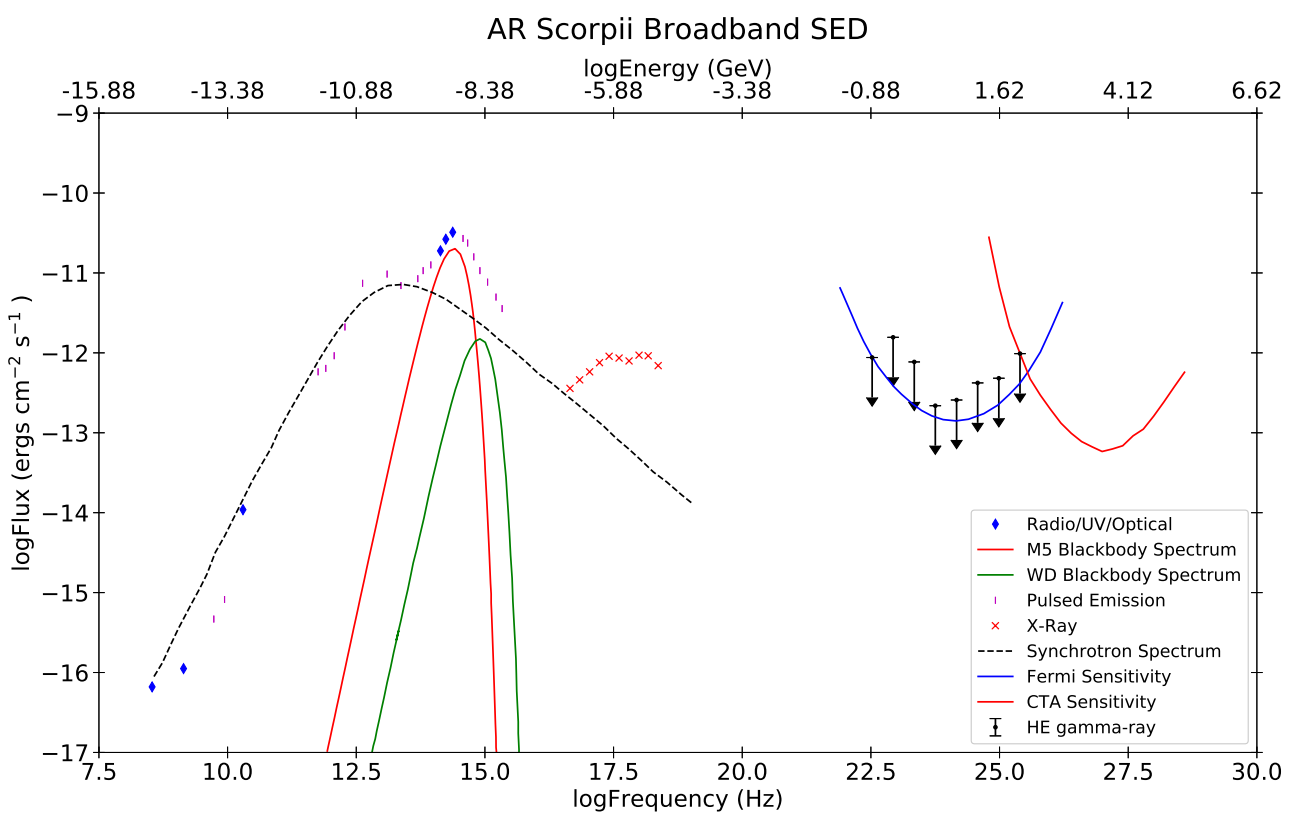

Figure 10: AR Sco broadband SED showing the gamma-ray upper limits in relation to the Fermi- LAT 10 year $^{1}$ (blue) and CTA-South 50 hour $^{2}$ (red) sensitivity curves.

${ }^{1}$ http : //www.slac.stanford.edu/exp/glast/groups/canda/lat-Performance.htm

${ }^{2}$ https : //portal.cta-observatory.org/CTA-Observatory/performance/SitePages/Home 


\section{Acknowledgements}

The authors thank Dr R.J. Britto for his guidance with the Fermi-LAT data analysis process.

\section{References}

[1] T. R. Marsh et al. A radio-pulsing white dwarf binary star, Nature 537 (2016) 374

[2] J.-J. Geng, B. Zhang, Y.-F. Huang A model of white dwarf pulsar AR Sco, Astrophys. J. Lett. 831 (2016) L1

[3] J.I. Katz AR Sco: Presenting a white dwarf synchronar, Astrophys. J. 835 (2017) 150

[4] D.A.H. Buckley, P.J. Meintjes, S.B. Potter, T.R. Marsh \& B.T. Gänsicke Polarimetric evidence of a white dwarf pulsar in the binary system AR Sco, Nature Astronomy 1 (2017) 0029

[5] D.A.H. Buckley, S.B Potter, P.J. Meintjes, T.R. Marsh and b B.T. Bänsicke Polarimetric evidence of the first white dwarf pulsar: The Binary System AR Scorpii, Galaxies 6 (2018) 14

[6] S.B. Potter et al. Polarized QPOs from the INTEGRAL polar IGRJ14536-5522 (= Swift J 1453.4 - 5524), MNRAS 402 (2010) 1161

[7] S.B. Potter and D.A.H. Buckley A re-evaluation of the proposed spin-down rate of the white dwarf pulsar in AR Scorpii, MNRAS 478 (2018) L78

[8] R.A. Stiller, C. Littlefield, P. Garnavich, C. Wood, F-J Hambsch and G. Meyers High-time resolution photometry of AR Scorpii: Confirmation of the white dwarf's spin-down, The Astronomical Journal 156(4) (2018) 150

[9] J. Takata, H Yang and k.S. Cheng A model for AR Scorpii: Emission from relativistic electrons trapped by by closed magnetic field lines of magnetic white dwarfs, The Astrophysical Journal 851 (2017) 143

[10] J. Takata, C. -P. Hu, L.C.C. Lin, P.H.T. Tam, P.S. Pal, C.Y. Hui, A.K.H Kong and K.S. Cheng A non-thermal pulsed X-ray emission of AR Scorpii, The Astrophysical Journal 853(2) (2018), 106

[11] W. Becker \& J. Trümper The X-ray luminosity of rotation powered neutron stars, Astronomy \& Astrophysics 326 (1997) 682

[12] V.S. Paliya et al. Detection of a Gamma-Ray Flare from the high-redshift Blazar DA 193, The Astrophysical Journal 871 (2019) 211

[13] S. Wilks The large sample distribution of the likelihood ratio for testing composite hypothesis, The Annals of Mathematical Statistics 9 (1938) 60

[14] J.R. Mattox et al. The likelihood analysis of EGRET data, The Astrophysical Journal 461 (1996) 396 